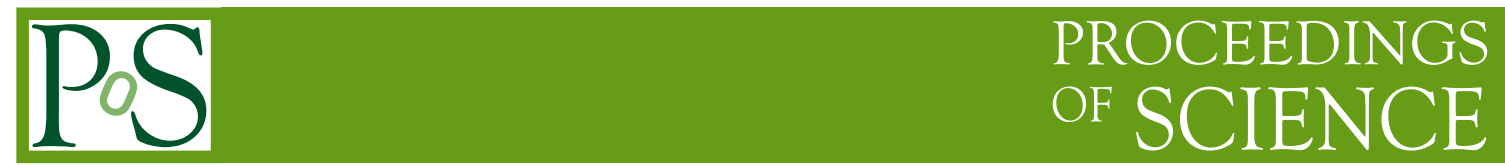

\title{
COMPASS facility beyond 2020
}

\section{Barbara Badelek ${ }^{* \dagger}$}

Faculty of Physics, University of Warsaw

E-mail: badelek@fuw.edu.pl

This paper summarizes the COMPASS Collaboration plans for its future beyond 2020, i.e. the programme planned between CERN accelerator long shutdowns 2 and 3: measurements using the muon beam on a transversely polarised deuteron target and proton radius measurement in the elastic muon-proton scattering. A hint is also given at the long-term programme, foreseen after the shutdown 3, with conventional muon and hadron beams as well as nonconventional, Radio Frequency-separated kaon and antiproton beams.

XXVI International Workshop on Deep-Inelastic Scattering and Related Subjects (DIS2018) 16-20 April 2018

Kobe, Japan

\footnotetext{
${ }^{*}$ Speaker.

${ }^{\dagger}$ On behalf of COMPASS Collaboration.
} 


\section{Introduction}

COMPASS facility [1] is very successful in studies of nucleon and other hadrons' structure and spectroscopy. It has taken data since 2002, with different beams and targets, see Table 1 . The COMPASS Collaboration, from 2014 onwards realising the programme called COMPASS II, has provided an impressive scientific output concerning the spin-dependent nucleon structure and the hadron spectroscopy. Ongoing are studies of Transverse Momentum Dependent Parton Distribution Functions (TMDs) as well as Generalised Parton Distributions (GPDs) which together provide an extension from the collinear to 3-dimensional picture of the nucleon and provide information on the parton orbital angular momentum there.

Planning of the future began in March 2016 with the "COMPASS beyond 2020 Workshop" at CERN, https://indico.cern.ch/event/502879/, intertwined with the CERN "Physics Beyond Colliders" initiative, https://indico.cern.ch/category/7885/. In this article the status of those (short- and long-term) plans will be highlighted.

Table 1: COMPASS data taking until now: dark red colour denotes DIS runs with polarised deuterium, while the red with polarised hydrogen.

\begin{tabular}{|l|l|}
\hline \hline $2002-2004$ & nucleon structure $\mu-\mathrm{d}, 160 \mathrm{GeV}, \mathrm{L}$ and T polarised target \\
2005 & CERN accelerator shutdown, increase of target acceptance \\
2006 & nucleon structure $\mu-\mathrm{d}, 160 \mathrm{GeV}, \mathrm{L}$ polarised target \\
2007 & nucleon structure $\mu-\mathrm{p}, 160 \mathrm{GeV}, \mathrm{L}$ and T polarised target \\
$2008-2009$ & hadron spectroscopy \\
2010 & nucleon structure $\mu-\mathrm{p}, 160 \mathrm{GeV}, \mathrm{T}$ polarised target \\
2011 & nucleon structure $\mu-\mathrm{p}, 200 \mathrm{GeV}, \mathrm{L}$ polarised target \\
2012 & Primakoff reaction $\left(\pi^{-}-\mathrm{C} / \mathrm{Ni} / \mathrm{W}\right), 190 \mathrm{GeV} ;$ \\
& DVCS/SIDIS $\mu-\mathrm{p}, 160 \mathrm{GeV}, \mathrm{unpolarised}$ target (test) \\
2013 & CERN accelerator shutdown, LS1 \\
2014 & Drell-Yan $\pi-\mathrm{p}$ reaction with T polarised target (test) \\
2015 & Drell-Yan $\pi-\mathrm{p}$ reaction with T polarised target \\
$2016-2017$ & DVCS/SIDIS $\mu-\mathrm{p}, 160 \mathrm{GeV}$, unpolarised target \\
2018 & Drell-Yan $\pi-\mathrm{p}$ reaction with T polarised target \\
$2019-2020$ & CERN accelerator shutdown, LS2 \\
\hline \hline After 2020: & This article \\
\hline \hline
\end{tabular}




\section{COMPASS Short Term Programme, [2]}

Since the CERN Research Board has approved in the memorandum DG-Dr-RCS-2017-093 an early post-LS2 fixed-target programme and running, the COMPASS-II Collaboration has decided to propose two physics cases of the future programme, as an addendum [2] to the ongoing programme, for data taking immediately after LS2.

The first programme, semi-inclusive DIS on transversely polarized deuterons, is the "missing piece" in the COMPASS data sets on transverse target spin orientations, see Table 1. In 2010, a dedicated run was taken on a transversely polarised proton $\left(\mathrm{NH}_{3}\right)$ target, which provided pioneering and unique information on the transversity and Sivers functions. On the contrary we provided only a marginal (albeit unique) data set for the isoscalar deuteron target. The older deuteron data have been taken only for short periods with the small-aperture target magnet so that the corresponding $\mathrm{d}$ data sets have 4 times less statistics than p sets, see Fig. 1.
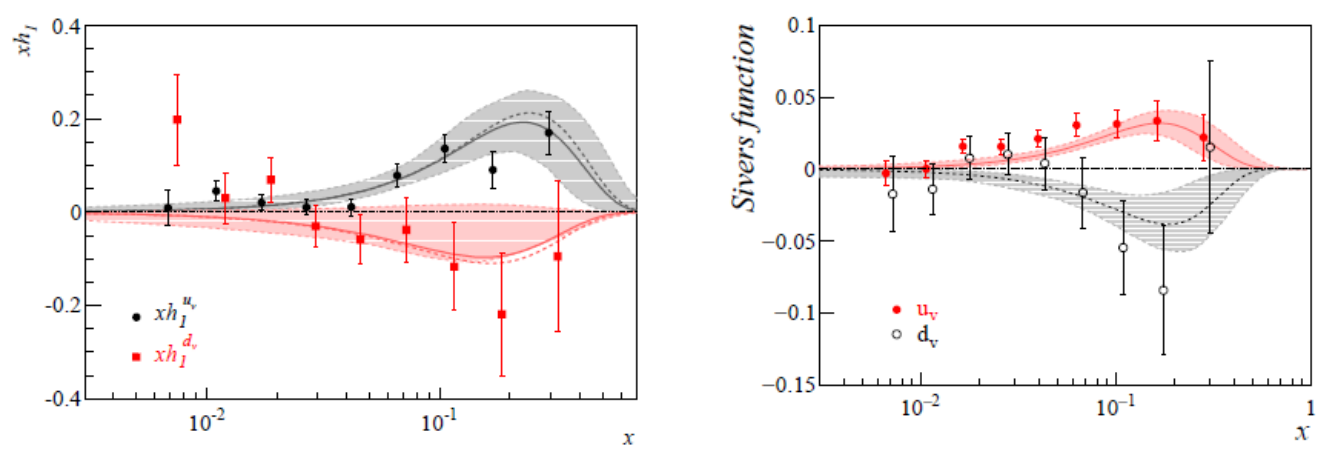

Figure 1: The transversity and Sivers PDFs extracted point-by-point using the existing COMPASS $\mathrm{p}$ and d data. The curves are the results of fits to the COMPASS and HERMES data and, for transversity, to the Belle data. Note that the uncertainty band for the d-quark transversity would be larger if the Soffer bound was not imposed. Figure from Ref. [2].

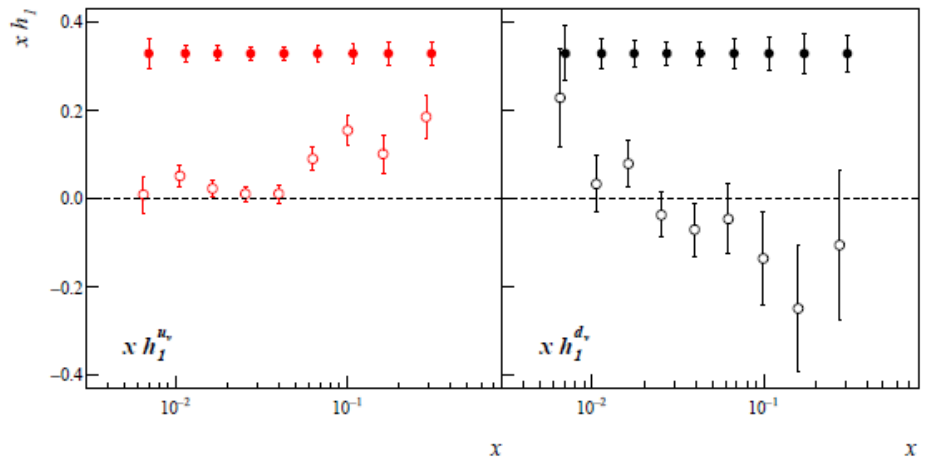

Figure 2: Values of the transversity for the $u_{v}$ (left) and $d_{v}$ quark (right) extracted from the existing $p$ and $d$ data (open circles), and the corresponding error bars estimated using the existing $\mathrm{p}$ data and the new data (closed circles). Figure from Ref. [2].

With one additional year of data taking, the statistical error of the deuteron measurements will be almost two times smaller than those of the corresponding proton data in all Bjorken- $x$ bins, Fig. 2 , 
allowing accurate flavour separation for the transversity and Sivers functions and measurements which will stay unique for many years to come. The latter encompass: determination of the full set of the Transverse Spin Asymmetries, TSAs, for the deuteron, extraction of the (truncated) nucleon tensor charge with accuracy two times better than now ( \pm 0.044 vs \pm 0.087 ), study of hard exclusive production of $\rho$ and $\omega$ mesons and finally a measurement of the $g_{2}$ function. Required apparatus upgrade is minimal and practically limited to the increase of the diameter of the target cells.

On 7th of June, 2018 the CERN Research Board has approved the COMPASS polarised deuteron run in 2021, i.e. immediately after the LS2.

The second programme, elastic muon-proton scattering, represents a new physics case for COMPASS. It was recognized recently that in the context of the currently debated "proton radius puzzle", where apparently the $(\mu, \mathrm{e})$ atomic data result in a different proton radii and some of them disagree with the electron scattering measurements [4], the muon-proton elastic scattering is a missing and complementary bit of the puzzle and a possible decisive experimental test. Details concerning this programme are given in the next section; here it should be mentioned that certain tests connected to this programme will be carried out parallely to the deuteron run in 2021.

\section{Long Term Programme, [3]}

The Long Term Programme is based on the COMPASS Collaboration experience gained in the past two decades on running the Hadron Spectroscopy, DIS/SIDIS, DVCS and Polarised Drell-Yan experiments. The upgraded COMPASS spectrometer with new elements, see Sec.3.7, will form a skeleton of the future QCD Facility. In this section selected Long Term programmes will shortly be mentioned.

\subsection{Proton radius measurement in $\mu \mathrm{p} \rightarrow \mu \mathrm{p}$ elastic scattering}

COMPASS-II is seen to be the ideal - in fact the only - place to realise the $\mu \mathrm{p} \rightarrow \mu \mathrm{p}$ experiment with multi-GeV muon beams. While a low energy $\mu$ p elastic scattering experiment, $\operatorname{MUSE}\left(E_{\mu} \lesssim\right.$ $0.5 \mathrm{GeV}$ ), is on the way [5] a high energy one may even be superior. Advantages of employing the high energy muon beam are: at high energies the Coulomb scattering angles are much smaller than at low energies and muon energy loss is negligible. It should be stressed that radiative corrections in case of the muon scattering are small and much smaller than for the electron beam. In the COMPASS measurements the reaction trigger will comprise a proton recoil (energies from about 0.5 to $100 \mathrm{MeV}$ ) and muon kink, yielding the cross section in the range $10^{-4} \lesssim Q^{2} /(\mathrm{GeV} / c)^{2} \lesssim 0.1$. An active high-pressure hydrogen TPC target IKAR will be employed; it was constructed by A. Vorobyev's group of St. Petersburg, who has developed a similar target for an experiment with electron beams at Mainz. The IKAR will possibly be supplemented by silicon fiber telescopes upand downstream the target. The goal of the measurement is $\Delta r_{E} \lesssim 0.01 \mathrm{fm}$ in 180 days beam time. This would need several new detector, readout and trigger developments.

\subsection{New Drell-Yan experiment with $190 \mathrm{GeV} \pi^{ \pm}$beams and $\mathrm{C}, \mathrm{W}$ targets}

Cross sections for the Drell-Yan process are very small; experiments should thus have very high luminosities; isoscalar, light targets are preferable; equipment acceptances should cope with possible large separation angles of the $\mu^{+} \mu^{-}$pair; as incident hadrons are separated from a beam, 
the particle identification is crucial; finally, a copious forward production of hadrons demands an active, very compact hadron absorber [7], possibly with W-Si detectors, which would have good tracking resolution, permitted a momentum measurement and had a large acceptance as $\theta_{\mu \mu} \gtrsim 250$ mrad.

Contrary to the nucleon, experimental information on the pion and kaon structure is scarce. The Drell-Yan and and J/ $\Psi$ production studies will be carried out with the $190 \mathrm{GeV} \pi^{ \pm}$beams and $\mathrm{C}$ and $\mathrm{W}$ targets. The valence and sea distributions for the pion will be determined; gluons will be extracted from direct $\gamma$ and charmonium production. Flavour dependent nuclear effects will be investigated provided a good beam charge balance and particle identification in the beams is provided.

\subsection{Exclusive reactions with high energy $\mu$ beam and transversely polarised proton target: GPD E measurement}

The GPDs describe the correlations between the longitudinal momentum and transverse spacial position of partons inside the nucleon; they permit accessing a contribution of the orbital angular momentum of partons to the nucleon spin. Asymmetries sensitive to the GPD E function may be measured with an accuracy of about $3 \%$ in 2 years of data taking. Compton form factors can be extracted in the interval, $0.01<x<0.1$, complementary to JLab/CLAS12. This interval has to be as large as possible if the total angular momentum carried by quarks in the nucleon has to be evaluated through the Ji sum rule [8]. Measurement would demand major modifications of the polarised target and building a suitable recoil detector.

\subsection{RF-separated hadron beams}

A newly designed and constructed Radio-Frequency separated hadron beams are the main challenge of the Long Term future programme. The idea is based on the old Panofsky-MontagueSchnell proposals, with two RF cavities [6]. Very preliminary estimates give probable values of 80 $\mathrm{GeV}$ and $110 \mathrm{GeV}$ momenta and intensity gains about 80 and 50 for kaon and antiproton beams, respectively. The latter should be compared with a $2.5 \%$ and $0.5 \%$ content of kaons and pions in a standard negative hadron beam.

\subsection{Kaon spectroscopy with kaon beam}

The goal here is to map out the complete spectrum of excited kaons with unprecedented precision and novel analysis methods. Samples of the order of 50 million events per year are foreseen with the RF-separated beam of at least $50 \mathrm{GeV}$ and uniform final state particle identification in a broad kinematic range. High precision vertex reconstruction and photon detection are experimental challenges here.

\subsection{Drell-Yan process with RF separated kaon and antiproton beams}

The overall gain in statistics here will be about a factor 50 to 100 compared to any previous DY experiments; data will be collected on the $\mathrm{NH}_{3}, \mathrm{Al}$ and $\mathrm{W}$ targets. For the kaon, the availability of $\mathrm{K}^{+}$and $K^{-}$beams will allow to disentangle valence and sea PDFs at small $x$. The gluon distributions can be accessed from direct $\gamma$ and $\mathrm{J} / \Psi$ productions. Measurements of TSAs with the $\overline{\mathrm{p}}$ beam will reduce the systematics of TMD PDFs of the proton. 


\subsection{Summary of all Long Term programmes}

Main features and hardware additions for Long Term programmes are summarised in Table 2.

Table 2: Requirements for future programs at the M2 beam line after 2021. Standard muon beams are in blue, standard hadron beams in green, and RF-separated hadron beams in red.

\begin{tabular}{|c|c|c|c|c|c|c|c|c|}
\hline Program & $\begin{array}{l}\text { Physics } \\
\text { Goals }\end{array}$ & $\begin{array}{c}\text { Beam } \\
\text { Energy } \\
{[\mathrm{GeV}]}\end{array}$ & $\begin{array}{c}\text { Beam } \\
\text { Intensity } \\
{\left[\mathrm{s}^{-1}\right]}\end{array}$ & $\begin{array}{c}\text { Trigger } \\
\text { Rate } \\
{[\mathrm{kHz}]}\end{array}$ & $\begin{array}{l}\text { Beam } \\
\text { Type }\end{array}$ & Target & $\begin{array}{c}\text { Earliest } \\
\text { start time, } \\
\text { duration }\end{array}$ & $\begin{array}{l}\text { Hardware } \\
\text { Additions }\end{array}$ \\
\hline $\begin{array}{c}\mu p \\
\text { elastic } \\
\text { scattering }\end{array}$ & $\begin{array}{c}\text { Precision } \\
\text { proton-radius } \\
\text { measurement }\end{array}$ & 100 & $4 \cdot 10^{6}$ & 100 & $\mu^{ \pm}$ & $\begin{array}{l}\text { high-pr. } \\
\text { H2 }\end{array}$ & $\begin{array}{l}2022 \\
1 \text { year }\end{array}$ & $\begin{array}{c}\text { active TPC } \\
\text { SciFi trigger } \\
\text { silicon veto }\end{array}$ \\
\hline $\begin{array}{c}\text { Hard } \\
\text { exclusive } \\
\text { reactions }\end{array}$ & GPD $E$ & 160 & $2 \cdot 10^{7}$ & 10 & $\mu^{ \pm}$ & $\mathrm{NH}_{3}^{\uparrow}$ & $\begin{array}{c}2022 \\
2 \text { years }\end{array}$ & $\begin{array}{c}\text { recoil silicon, } \\
\text { modified } \\
\text { PT magnet }\end{array}$ \\
\hline $\begin{array}{l}\text { Input for } \\
\text { DMS }\end{array}$ & $\begin{array}{l}\bar{p} \text { production } \\
\text { cross-section }\end{array}$ & $20-280$ & $5 \cdot 10^{5}$ & 25 & $p$ & $\begin{array}{l}\text { LH2, } \\
\text { LHe }\end{array}$ & $\begin{array}{c}2022 \\
1 \text { month }\end{array}$ & $\begin{array}{l}\text { LHe } \\
\text { target }\end{array}$ \\
\hline $\begin{array}{c}\bar{p} \text {-induced } \\
\text { Spectroscopy }\end{array}$ & $\begin{array}{c}\text { Heavy quark } \\
\text { exotics }\end{array}$ & 12,20 & $5 \cdot 10^{7}$ & 25 & $\bar{p}$ & LH2 & $\begin{array}{c}2022 \\
2 \text { years }\end{array}$ & $\begin{array}{c}\text { target spectr.: } \\
\text { tracking, } \\
\text { calorimetry }\end{array}$ \\
\hline Drell-Yan & Pion PDFs & 190 & $7 \cdot 10^{7}$ & 25 & $\pi^{ \pm}$ & $\mathrm{C} / \mathrm{W}$ & $\begin{array}{c}2022 \\
1-2 \text { years }\end{array}$ & \\
\hline $\begin{array}{l}\text { Drell-Yan } \\
\quad(\mathrm{RF})\end{array}$ & $\begin{array}{c}\text { Kaon PDFs } \\
\text { Nucleon TMDs }\end{array}$ & $\sim 100$ & $10^{8}$ & $25-50$ & $K^{ \pm}, \bar{p}$ & $\begin{array}{l}\mathrm{NH}_{3}^{\uparrow}, \\
\mathrm{C} / \mathrm{W}\end{array}$ & $\begin{array}{c}2026 \\
2-3 \text { years }\end{array}$ & $\begin{array}{c}\text { "active } \\
\text { absorber", } \\
\text { vertex det. }\end{array}$ \\
\hline $\begin{array}{l}\text { Primakoff } \\
(\mathrm{RF})\end{array}$ & $\begin{array}{c}\text { Kaon polarizi- } \\
\text { bility \& pion } \\
\text { life time }\end{array}$ & $\sim 100$ & $5 \cdot 10^{6}$ & $>10$ & $K^{-}$ & $\mathrm{Ni}$ & $\begin{array}{c}\text { non-exclusive } \\
2026 \\
1 \text { year }\end{array}$ & \\
\hline $\begin{array}{c}\text { Prompt } \\
\text { Photons } \\
\text { (RF) }\end{array}$ & $\begin{array}{l}\text { Meson gluon } \\
\text { PDFs }\end{array}$ & $\geq 100$ & $5 \cdot 10^{6}$ & $10-100$ & $\begin{array}{l}K^{ \pm} \\
\pi^{ \pm} \\
\end{array}$ & $\begin{array}{c}\mathrm{LH} 2, \\
\mathrm{Ni}\end{array}$ & $\begin{array}{c}\text { non-exclusive } \\
2026 \\
1-2 \text { years }\end{array}$ & hodoscope \\
\hline $\begin{array}{c}K \text {-induced } \\
\text { Spectroscopy } \\
(\mathrm{RF})\end{array}$ & $\begin{array}{c}\text { High-precision } \\
\text { strange-meson } \\
\text { spectrum } \\
\end{array}$ & $50-100$ & $5 \cdot 10^{6}$ & 25 & $K^{-}$ & LH2 & $\begin{array}{l}2026 \\
1 \text { year }\end{array}$ & $\begin{array}{l}\text { recoil TOF } \\
\text { forward } \\
\text { PID }\end{array}$ \\
\hline $\begin{array}{l}\text { Vector mesons } \\
\text { (RF) }\end{array}$ & $\begin{array}{c}\text { Spin Density } \\
\text { Matrix } \\
\text { Elements }\end{array}$ & $50-100$ & $5 \cdot 10^{6}$ & $10-100$ & $K^{ \pm}, \pi^{ \pm}$ & $\begin{array}{c}\text { from } \mathrm{H} \\
\text { to } \mathrm{Pb}\end{array}$ & $\begin{array}{l}2026 \\
1 \text { year }\end{array}$ & \\
\hline
\end{tabular}

\section{References}

[1] COMPASS Collaboration, P. Abbon et al., Nucl. Instrum. Meth. A 577 (2007) 455.

[2] COMPASS Collaboration, K. Augsten et al., CERN-SPSC-2017-034

[3] “A New QCD facility at the M2 beam line beyond 2021" - Letter of Intent, to appear (2018).

[4] See e.g. A. Beyer et al., Science, 358 (2017) 79; W. Vassen, ibid p.39; H. Fleurbaey et al., Phys. Rev. Lett. 120 (2018) 183001.

[5] MUSE Collaboration, R. Gilman et al., arXiv:1303.2160.

[6] P. Bernard et al., CERN 68-29.

[7] See e.g. M. Antonova et al., arXiv:1704.08079.

[8] X. -D. Ji, Phys. Rev. Lett. 78 (1997) 610. 